\title{
Gliomas: casuística cirúrgica em um hospital-escola quaternário
}

\author{
Clemar Correa ${ }^{1}$, Matheus Schmidt Soares², Hector Tomas Navarro Cabrera', \\ Eberval Gadelha Figueiredo ${ }^{3}$, Manoel Jacobsen Teixeira ${ }^{4}$ \\ Divisão de Clínica Neurocirúrgica do HCFMUSP
}

\section{RESUMO}

Objetivo: É apresentada neste trabalho a casuística cirúrgica de ressecção de gliomas em um hospitalescola quaternário da cidade de São Paulo, no período de 19 meses. Método: Foram coletados dados, prospectivamente, de todos os pacientes adultos com diagnóstico de glioma, submetidos à ressecção cirúrgica no hospital referido, no período de novembro de 2007 a junho de 2009, por meio de preenchimento de um protocolo pelo médico-assistente de cada caso. Resultados: No período estudado, foram realizadas 288 cirurgias para ressecção de tumores encefálicos. Dessas, 88 cirurgias foram destinadas à ressecção de gliomas do sistema nervoso central. Corresponde a $30,56 \%$ das cirurgias para ressecção de tumores encefálicos, sendo esse o maior grupo, com média anual de 55,5 gliomas operados. Conclusão: O predomínio dos astrocitomas de alto grau nos casos operados é semelhante aos dados da literatura, demonstrando a importância dessa doença. A gravidade e a considerável incidência desses tumores em nossa população mostram a necessidade de constante investimento e aprimoramento tecnológico e humano para o adequado tratamento desses pacientes.

\section{PALAVRAS-CHAVE}

Glioma, astrocitoma, glioblastoma, neurocirurgia, neoplasias encefálicas.

\section{ABSTRACT}

Gliomas: a quaternary medical school hospital surgical casuistic

Objective: We present in this work the gliomas surgery casuistic of a quaternary medical school hospital of São Paulo city during a 19 months period. Method: Data were collected, prospectively, of all patients with glioma operated at Hospital das Clínicas of São Paulo Medical School, in the period between November 2007 to June 2009. Results: In the studied period 288 surgeries were held for encephalic tumors resection. Of these, 88 surgeries were aimed at resection of central nervous system gliomas. That represents $30.56 \%$ of encephalic tumors surgery, the largest group with an annual average of 55.5 gliomas operated. Conclusion: The prevalence of high grade astrocytomas operated is similar to literature data, demonstrating the importance of this disease on the health of a community. The seriousness and considerable incidence of these tumors in our population show the necessity of constant investment in technological and human enhancement for the proper treatment of these patients.

\section{KEYWORDS}

Glioma, astrocytoma, glioblastoma, neurosurgery, brain neoplasms.

1. Médico-assistente da Divisão de Neurocirurgia do Hospital das Clínicas da Faculdade de Medicina da Universidade de São Paulo (HCFMUSP).

2. Médico preceptor dos residentes de Neurocirurgia do HCFMUSP.

3. Médico supervisor da Divisão de Neurocirurgia do HCFMUSP.

4. Professor titular da disciplina de Neurocirurgia da FMUSP. 


\section{Introdução}

Glioma é um termo referente a qualquer neoplasia derivada de células gliais e compreende astrocitomas, oligodendrogliomas, ependimomas e tumores derivados do plexo coroide. Gliomas infiltrativos são os tumores primários mais comuns do sistema nervoso central (SNC), sendo $40 \%$ dos tumores primários e $78 \%$ dentre os tumores malignos que acometem o SNC. ${ }^{1}$ Mais de $80 \%$ desses tumores são considerados gliomas de alto grau (graus III e IV) quando classificados de acordo com os critérios da OMS, ${ }^{2,3}$ sistema baseado na diferenciação morfológica das linhagens astrocíticas, oligodendrogliais e mistas, além de graduação de malignidade conforme características histológicas.

Os sistemas de classificação atuais se baseiam nos trabalhos realizados por Baley e Cushing em 1926', que classificavam os tumores de acordo com os tipos celulares do SNC que mais se assemelhavam às células tumorais. A classificação e a graduação dos gliomas vêm sendo redefinidas periodicamente ao longo desses 80 anos, por meio dos esquemas de classificação de Baley e Cushing, Kernohan, Ringertz, Nelson, St Anne-Mayo e OMS. ${ }^{5}$

Apresentamos neste trabalho a casuística cirúrgica de gliomas em um hospital-escola quaternário da cidade de São Paulo, no período de novembro de 2007 a junho de 2009, totalizando 19 meses.

\section{Método}

Foram coletados dados, prospectivamente, de todos os gliomas operados em adultos no Hospital das
Clínicas da Faculdade de Medicina da Universidade de São Paulo, no período de 19 meses entre novembro de 2007 e junho de 2009. Foram excluídas dessa casuística todas as cirurgias que não foram relacionadas à ressecção dos gliomas, como derivações ventrículo-peritoniais, cranioplastias e limpezas cirúrgicas. Os tumores foram divididos por diagnóstico, idade de acometimento da doença, complicações pós-operatórias e sobrevida média.

\section{Resultados}

No período estudado, foram realizadas 288 cirurgias para ressecção de tumores encefálicos (Figura 1). Destas, 88 cirurgias foram destinadas à ressecção de gliomas do SNC, o que corresponde a $30,56 \%$ das cirurgias para ressecção de tumores encefálicos, sendo esse o maior grupo, e uma média anual de 55,5 gliomas operados. $\mathrm{O}$ segundo maior grupo de pacientes operados foi composto pelas metástases, com 68 casos $(23,61 \%)$, seguidas pelos meningeomas, com 59 casos (20,49\%). Dos 88 gliomas operados (Tabela 1), 46 casos correspondiam a GBM e 9 casos, a astrocitomas anaplásicos, o que representa $63 \%$ de gliomas de alto grau (III e IV). O restante é composto por 14 oligodendrogliomas (16\%), 7 astrocitomas grau II (8\%), 5 oligoastrocitomas (5,6\%), 3 gangliogliomas $(3,4 \%)$, entre outros mais raros $(4,5 \%)$.

$\mathrm{Na}$ evolução desses 88 casos operados, houve 3 casos de infecções pós-operatórias (3,4\%), 4 sangramentos em leito cirúrgico $(4,5 \%), 2$ casos com piora importante do edema cerebral pós-operatório $(2,2 \%)$ e 1 caso de fístula liquórica incisional $(1,1 \%)$, totalizando 10 complicações pós-operatórias $(11,3 \%)$.
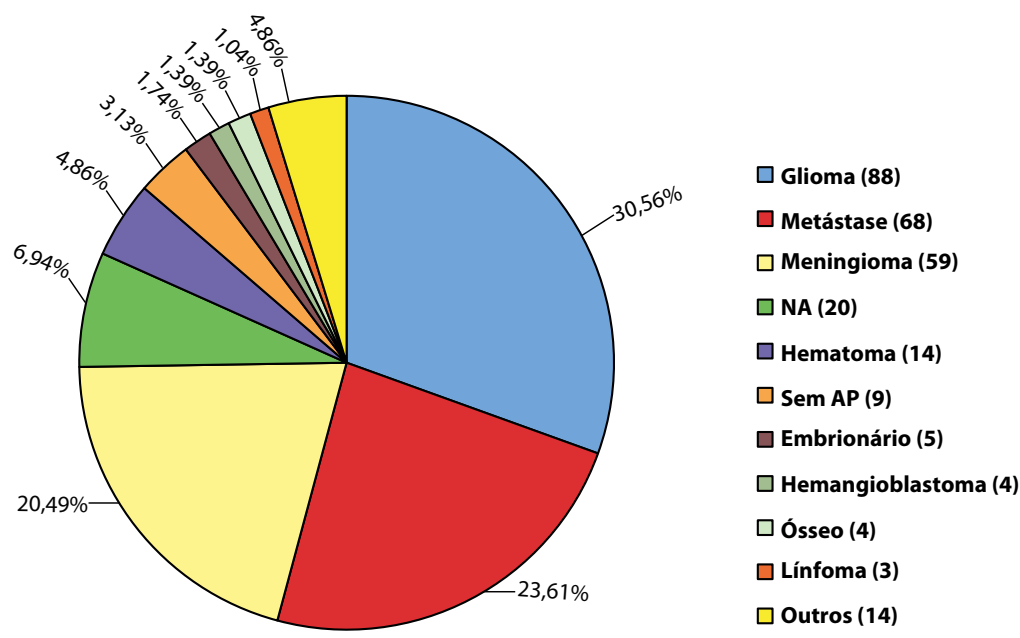

Figura 1 - Distribuição por diagnóstico. 


\begin{tabular}{|c|c|c|c|c|}
\hline Tipo de glioma & Número de casos & Média anual & $\begin{array}{l}\text { Complicações: Sangramento, } \\
\text { infecção, fístula, inchaço }\end{array}$ & Porcentagem de complicações cirúrgicas \\
\hline GBM & 46 & 29,89 & $\begin{array}{l}3 \text { infecções, } 2 \text { sangramentos, } \\
\text { reop } 1 \text { inchaço, sem reop }\end{array}$ & $13,04 \%$ \\
\hline Olidendrogliomas & 14 & 9,73 & 1 , sangramento reop 1, fístula reop & $7,14 \%$ \\
\hline Astro II & 07 & 4,86 & 1, inchaço, reop & $14,28 \%$ \\
\hline Astro III & 09 & 4,17 & 1, sangramento, reop & $11,1 \%$ \\
\hline Oligoastrocitomas & 05 & 3,47 & 0 & 0 \\
\hline Gangliogliomas & 03 & 2,08 & 0 & 0 \\
\hline Astro I & 01 & 0,69 & 0 & 0 \\
\hline Neurocitomas & 01 & 0,69 & 0 & 0 \\
\hline DNET & 01 & 0,69 & 0 & 0 \\
\hline Ependimomas & 01 & 0,69 & 0 & 0 \\
\hline Total & 88 & 57,00 & 10 & $11,3 \%$ \\
\hline
\end{tabular}

\section{Discussão}

A classificação dos tumores do SNC é motivo de contínuo debate. Atualmente, o sistema mais utilizado e difundido mundialmente é o sistema da OMS, que teve sua primeira edição realizada em 1979. Esse sistema prevê quatro graus ascendentes de malignidade. Tumores grau I têm comportamento biológico e histológico benignos, sendo quase sempre curados após ressecção cirúrgica total. Estão incluídos nesse grau os astrocitomas pilocíticos, os astrocitomas subependimários de células gigantes e os papilomas de plexo coroide. A transformação maligna e a recorrência desses tumores são eventos raros. Os tumores graduados de II a IV têm comportamento histológico e biológico malignos e são classificados conforme critérios histológicos bem definidos. São considerados grau II os astrocitomas difusos, os xantoastrocitomas pleomórficos, os oligodendrogliomas, os oligoastrocitomas, os ependimomas e os papilomas atípicos. No grau III estão os astrocitomas anaplásicos, que se diferenciam dos de grau II, entre outros, pelo grau de atividade mitótica celular. A média de sobrevida para esses tumores grau III varia entre $3 \mathrm{e}$ 5 anos. Os tumores grau IV são os glioblastomas multiformes (GBM), que se diferenciam dos de grau III pela proliferação neovascular e necrose, e têm uma média de sobrevida de aproximadamente 1 ano. ${ }^{2,3,5}$

Os astrocitomas de grau II (baixo grau) representam um grupo heterogêneo de tumores e correspondem a $11 \%$ de todas as neoplasias primárias do sistema nervoso central (SNC). Eles acometem predominantemente adultos entre 20 e 40 anos e, ao contrário dos gliomas de alto grau, sua incidência diminui com a idade, sendo raros após os 50 anos. São neoplasias malignas, porém de comportamento mais indolente. $\mathrm{O}$ fato de poder haver evolução lenta e praticamente assintomática por muitos anos torna o tratamento controverso. A observação após biópsia, a ressecção cirúrgica agressiva e a radioterapia fracionada são opções de condutas, porém não obrigatórias. Estudos genéticos e moleculares podem auxiliar na decisão terapêutica, como a presença da codeleção cromossômica $1 \mathrm{p} 19 \mathrm{q}$ em oligodendrogliomas, que favorece o tratamento quimioterápico. ${ }^{6-8}$

Os gliomas de alto grau, grau III e IV, constituem $77 \%$ dos casos de tumores malignos do SNC, sendo, desses, $82 \%$ representados por GBM. ${ }^{9}$ A incidência é maior entre 65 e 75 anos e homens são mais afetados que mulheres. A sobrevida mediana diminui com o avanço da idade. Os fatores prognósticos mais importantes que interferem na resposta ao tratamento são idade, grau tumoral (astrocitoma anaplásico x GBM) e a performance no Karnosfky performance status (KPS). O tratamento-padrão para esses pacientes consiste em ressecção cirúrgica seguida de radioterapia e quimioterapia adjuvante..$^{10-12}$

Apresentamos aqui a casuística cirúrgica de tumores encefálicos de um hospital-escola quaternário. O predomínio dos astrocitomas de alto grau nos casos operados é semelhante aos dados da literatura, demonstrando a importância dessa doença. Nos gliomas de baixo grau, nesta casuística, percebe-se o predomínio de oligodendrogliomas em relação aos astrocitomas difusos. Isso se deve, provavelmente, ao maior número de indicações de ressecção cirúrgica dos oligodendrogliomas, considerando sua boa resposta ao tratamento cirúrgico seguido de tratamento adjuvante. A mortalidade cirúrgica foi nula e o número de complicações graves em nossa instituição foi baixo. A gravidade e a considerável incidência desses tumores em nossa população mostram a necessidade de constante investimento e aprimoramento tecnológico e humano para o adequado tratamento desses pacientes. 


\section{Referências}

1. Central Brain Tumor Registry of the United States: Statistical Report: Primary Brain Tumors in the United States, 1998-2002. Hinsdale, III: Central Brain Tumor Registry of the United States; 2006.

2. Kleihues P, Cavenee WK. Pathology and genetics of tumours of the nervous system. Lyon, France: IARC Press; 2000. p. 314. World Health Organization Classification of Tumours.

3. Zulch KJ. Histological typing of tumors of the central nervous system. Geneva, Switzerland: World Health Organization; 1979.

4. Bailey $\mathrm{P}$, Cushing $\mathrm{H}$. A classification of tumours of the glioma group on a histogenetic basis with a correlated study of prognosis. Philadelphia: JB Lippincott; 1926.

5. Miller CR, Perry A. Glioblastoma, morphological and molecular diversity. Arch Pathol Lab Med. 2007;131:397-406.

6. Kreth FW, Faist M, Rossner R, et al. Supratentorial World Health Organization grade 2 astrocytomas and oligostrocytomas: a new pattern of prognostic factors. Cancer. 1997;79:370-9.

7. Janny P, Cure H, Mohr M, Heldt N, Kwiatkowski F, Lemaire JJ, et al. Low grade supratentorial astrocytomas: management and prognostic factors. Cancer. 1994;73:1937-45.
8. Smith JS, Perry A, Borell TJ, Lee HK, O'Fallon J, Hosek $\mathrm{SM}$, et al. Alterations of chromosome arms $1 p$ and $19 q$ as predictors of survival in oligodendrogliomas, astrocytomas and mixed oligoastrocytomas. J Clin Oncol. 2000;18:636-45.

9. Brandes AA. State of the art treatment of high grade brain tumors. Semin Oncol. 2003;30(6 Suppl 19):4-9.

10. Grossman SA, Batara JF. Current management of glioblastoma multiforme. Semin Oncol. 2004;31:635-44.

11. Brandes AA, Compostella A, Blatt V. Glioblastoma in the elderly: current and future trends. Crit Rev Oncol Hematol. 2006;60:256-66.

12. DeAngelis LM, Burger PC, Green SB. Malignant glioma: who benefits from adjuvant chemotherapy? Ann Neurol. 1998;44:691-5.

Endereço para correspondência

Matheus Schmidt Soares

Rua Alves Guimarães, 642, ap. 33, Pinheiros

05410-001 - São Paulo, SP

E-mail: matheus_med@yahoo.com.br 